\title{
Large back-angle quasielastic scattering for ${ }^{7} \mathrm{Li}+{ }^{159} \mathrm{~Tb}$
}

\author{
Piyasi Biswas $^{1,3 \dagger}$, A. Mukherjee ${ }^{1,3} *$ D. Chattopadhyay ${ }^{1}$, Saikat Bhattacharjee ${ }^{1,3}$, M.K. Pradhan ${ }^{1 \ddagger}$, Md. Moin $^{2}$ \\ Shaikh $^{1 \S}$, Subinit Roy ${ }^{1,3}$, A. Goswami ${ }^{1 \uparrow}$, P. Basu ${ }^{1 * *}$, S. Santra ${ }^{2,3}$, S.K. Pandit ${ }^{2,3}$, K. Mahata ${ }^{2,3}$, and A. Shrivastava ${ }^{2,3}$ \\ ${ }^{1}$ Saha Institute of Nuclear Physics, 1/AF Bidhannagar, Kolkata-700064, India \\ ${ }^{2}$ Nuclear Physics Division, Bhabha Atomic Research Centre, Mumbai-400085, India \\ ${ }^{3}$ Homi Bhabha National Institute, Anushaktinagar, Mumbai-400094, India
}

(Dated: January 11, 2021)

\begin{abstract}
Quasielastic scattering excitation function at large backward angle has been measured for the weakly bound system, ${ }^{7} \mathrm{Li}+{ }^{159} \mathrm{~Tb}$ at energies around the Coulomb barrier. The corresponding quasielastic barrier distribution has been derived from the excitation function, both including and excluding the $\alpha$-particles produced in the reaction. The centroid of the barrier distribution obtained after inclusion of $\alpha$-particles was found to be shifted higher in energy, compared to the distribution excluding the $\alpha$-particles. The quasielastic data, excluding the $\alpha$-particles, have been analyzed in the framework of continuum discretized coupled channel calculations. The quasielastic barrier distribution for ${ }^{7} \mathrm{Li}+{ }^{159} \mathrm{~Tb}$, has also been compared with the fusion barrier distribution for the system.
\end{abstract}

\section{INTRODUCTION}

Heavy ion fusion at near-barrier energies is strongly affected by the internal structure of the colliding nuclei and coupling to the direct nuclear processes, like inelastic excitation and direct nucleon transfer. The coupling of the relative motion to the internal degrees of freedom successfully explained the sub-barrier fusion enhancement observed in heavy ion collisions with respect to the one dimensional barrier penetration model calculations [1, 2].

The coupling essentially modifies the effective interaction potential and in turn splits the single, uncoupled fusion barrier into a distribution of barriers. The fusion barrier distribution, $D_{\text {fus }}$ for a system can be derived from the measured fusion excitation function as [3]

$$
D_{f u s}(E)=\frac{d^{2}}{d E^{2}}\left\lfloor E \sigma_{f u s}(E)\right\rfloor
$$

where $\sigma_{f u s}(E)$ is the fusion cross section for the system at the center-of-mass energy, E. Over the past several years of research in heavy ion collision, $D_{f u s}(E)$ has evolved to be a powerful tool to decipher the effects of coupling of various channels on sub-barrier fusion and hence probe the reaction dynamics of nucleus-nucleus collision [2]. Since extraction of $D_{f u s}(E)$ involves second derivative of $E \sigma_{f u s}(E)$, obtaining a meaningful barrier distribution requires very precisely measured fusion data.

\footnotetext{
${ }^{\dagger}$ Present address:Shahid Matangini Hazra Government College for Women, Tamluk, Chakshrikrishnapur, Kulberia, Purba Medinipur, West Bengal - 721649, India

${ }^{\ddagger}$ Present address: Department of Physics, Belda College, Belda, Paschim Medinipur, West Bengal - 721424, India

$\S$ Present address: Department of Physics, Chanchal College, Chanchal, Malda, West Bengal - 732123, India

IDeceased

** Retired

*Electronic address: anjali.mukherjee@saha.ac.in
}

A similar barrier distribution can also be extracted from large back-angle quasielastic scattering excitation function [4]. The quasielastic scattering is defined as the sum of all direct processes, like elastic and inelastic scattering and transfer processes. Fusion is related to transmission through the barrier, whereas large backangle quasielastic scattering is related to reflection at the barrier. Because of the conservation of reaction flux, these two processes may be considered as complementary to each other. The quasielastic barrier distribution, $D_{q e l}$ is obtained as [4],

$$
D_{q e l}(E)=-\frac{d}{d E}\left\lfloor\frac{d \sigma_{q e l}}{d \sigma_{\text {Ruth }}}(E)\right\rfloor
$$

where $\left(d \sigma_{q e l} / d \sigma_{R u t h}\right)$ is the ratio of quasielastic scattering and Rutherford scattering differential cross sections at a fixed back-angle. As $D_{q e l}$ is derived from the first derivative, unlike $D_{\text {fus }}$, the uncertainty associated with $D_{q e l}$ is less than that associated with $D_{\text {fus }}$.

It has been observed that for heavy ion collisions involving tightly bound nuclei, where fusion is the most dominant reaction process at near-barrier energies, $D_{\text {fus }}$ and $D_{q e l}$ are very similar [2, 5- 8$]$. By contrast, for very heavy systems, where deep inelastic processes become important, it has been argued by Zagrabaev [9] that the quasielastic barrier distribution extracted from the sum of elastic and inelastic backscattering processes represents the total reaction threshold distribution and it differs from the distribution derived from the fusion excitation function. For reactions, where cross sections of non-fusion channels are comparable to fusion cross sections, a deviation of $D_{q e l}$ from $D_{f u s}$ is expected to be seen [10, 11].

Similarly, for weakly bound systems the distribution $D_{\text {qel }}$ extracted only from the sum of the contribution of elastic, inelastic and transfer processes at large backangle will provide information about the total reaction threshold distribution and not about the fusion barriers. This is because of the fact that for weakly bound systems, apart from other direct processes, breakup, 
transfer induced breakup and incomplete fusion (ICF) are very important processes competing with fusion at near-barrier energies. Several experimental studies of quasielastic scattering excitation function at large backangle and corresponding barrier distribution have been reported for various systems involving weakly bound stable projectiles [8, 12 22]. In most of these works, the measured quasielastic scattering excitation function and the corresponding $D_{q e l}$ were analyzed within the framework of coupled reaction channel (CRC) or continuum discretized coupled channel (CDCC) models, while a few of these works compared $D_{\text {qel }}$ with $D_{\text {fus }}$. The latter works showed that for weakly bound systems, $D_{q e l}$ is in general broader compared to $D_{f u s}$. Also, the centroid of the distribution $D_{q e l}$, with quasielastic events defined as the sum of elastic and inelastic scattering and transfer processes, is found to be shifted lower in energy than that of $D_{\text {fus }}$. But the shift in energy between the centroids of $D_{\text {qel }}$ and $D_{\text {fus }}$ is found to be different for different systems. For ${ }^{6,7} \mathrm{Li}$ induced reactions with ${ }^{64} \mathrm{Ni}(\mathrm{Z}=28)$, though the peak of $D_{q e l}$ is observed to be shifted towards lower energy compared to $D_{\text {fus }}$ for ${ }^{6} \mathrm{Li}+{ }^{64} \mathrm{Ni}$ [21], the distributions $D_{q e l}$ and $D_{\text {fus }}$ are seen to be similar for ${ }^{7} \mathrm{Li}+{ }^{64} \mathrm{Ni}$ 22. . For ${ }^{6,7} \mathrm{Li}$ induced reactions with ${ }^{208} \mathrm{~Pb}$ ( $\mathrm{Z}=82)$ [8], $D_{\text {qel }}$ and $D_{\text {fus }}$ have been reported to be similar if breakup contribution is included in the quasielastic scattering excitation function. However, for ${ }^{6,7} \mathrm{Li}$ induced reactions with ${ }^{197} \mathrm{Au}(\mathrm{Z}=79)$ [20], distributions $D_{\text {qel }}$ are seen to shift towards higher energies with respect to $D_{\text {fus }}$ after inclusion of breakup- $\alpha$ channel in the quasielastic scattering excitation functions for both ${ }^{6} \mathrm{Li}$ and ${ }^{7} \mathrm{Li}$ cases. The observations reported for ${ }^{6,7} \mathrm{Li}$-induced reactions with ${ }^{208} \mathrm{~Pb}$ and ${ }^{197} \mathrm{Au}$ are contradictory, though ${ }^{197} \mathrm{Au}$ nucleus lies very close to ${ }^{208} \mathrm{~Pb}$. In the backdrop of this scenario, it would be interesting to investigate the role of the structure of target nuclei while comparing $D_{q e l}$ with $D_{\text {fus }}$ in ${ }^{6,7} \mathrm{Li}$-induced reactions.

In this context, we carried out a measurement of large back-angle quasielastic excitation function and the corresponding barrier distribution for the system ${ }^{7} \mathrm{Li}+{ }^{159} \mathrm{~Tb}$, at near-barrier energies, where ${ }^{159} \mathrm{~Tb}$ is a well-deformed target nucleus. A preliminary analysis of these measurements was reported in Ref. 23]. This work is a part of our systematic investigation of different reaction mechanisms in ${ }^{6,7} \mathrm{Li}+{ }^{159} \mathrm{~Tb}[24-26]$. Complete and incomplete fusion excitation functions for ${ }^{6,7} \mathrm{Li}+{ }^{159} \mathrm{~Tb}$ were reported in Refs. 24, 25]. Different processes contributing to the measured large $\alpha$-yield in the reaction ${ }^{6} \mathrm{Li}+{ }^{159} \mathrm{~Tb}$ were disentangled and reported in Ref. [26]. The primary motivation of the present work is to investigate the role of couplings to ${ }^{7} \mathrm{Li}$ projectile and ${ }^{159} \mathrm{~Tb}$ target excitations on large back-angle quasielastic scattering process within the framework of coupled channel calculations.

The present paper is organized as follows: The experimental details, along with the results are described in Sec. II. The measured quasielastic scattering excitation function and the corresponding barrier distribution are analyzed in the framework of coupled channel calcula- tions in Sec. III. A comparison of the $D_{q e l}$ and $D_{\text {fus }}$ is discussed in Sec. IV. Finally, Sec. V summarizes the work.

\section{EXPERIMENTAL DETAILS}

\section{II.I. Experimental setup}

The ${ }^{7} \mathrm{Li}$ beams in the energy range $17-34 \mathrm{MeV}$, in steps of $1 \mathrm{MeV}$, from the 14UD BARC-TIFR Pelletron Accelerator at Mumbai, India were used to bombard a selfsupporting ${ }^{159} \mathrm{~Tb}$ target foil of thickness $\approx 1.1 \mathrm{mg} / \mathrm{cm}^{2}$. The energies of the incident beam were corrected for the loss of energy in the target material at half-thickness of the target. To detect and identify the charged particles produced in the reaction, a set of four $\Delta \mathrm{E}-\mathrm{E}$ telescopes of Si-surface barrier detectors were placed at $\pm 170^{\circ}$ and $\pm 160^{\circ}$ inside a scattering chamber of diameter $1 \mathrm{~m}$. The $\Delta \mathrm{E}$-E telescopes were mounted at $\pm 170^{\circ}$ and $\pm 160^{\circ}$ primarily to check the consistency of the measured quasielastic scattering events. The thicknesses of the detectors of each telescope were so chosen that the charged particles lose part of their kinetic energies in the first detector $(\Delta \mathrm{E})$ and stop by depositing the residual energies $\left(\mathrm{E}_{r e s}\right)$ in the second detector (E). However, the stop detectors used in the experiment were not thick enough to stop the $\mathrm{Z}=1$ particles. Two Si-surface barrier detectors, of thicknesses $300 \mu \mathrm{m}$ and $500 \mu \mathrm{m}$, were placed at $\pm 20^{\circ}$ with respect to the beam direction for monitoring the beam and also for normalization purposes. In front of each telescope and monitor, a collimator was placed to define the solid angle.

\section{II.II. Data analysis and Results}

A typical two-dimensional $\Delta \mathrm{E}^{-\mathrm{E}_{t o t}}$ (where, $\mathrm{E}_{\text {tot }}=\Delta \mathrm{E}+\mathrm{E}_{\text {res }}$ ) spectrum, at $E_{l a b}=26 \mathrm{MeV}$ is shown in Fig. 1 The peak in the $\mathrm{Z}=3$ band arises from contributions due to elastic scattering of ${ }^{7} \mathrm{Li}$ and inelastic scattering from the excited states of ${ }^{159} \mathrm{~Tb}$. The low-lying levels of ${ }^{159} \mathrm{~Tb}$ are very closely spaced and so the inelastic excited states of the target could not be separated from the elastic events. Besides, the $\mathrm{Z}=3$ band may also contain contribution from the inelastic excitation of the projectile, ${ }^{7} \mathrm{Li}$ [19]. Moreover, it may also contain contribution from ${ }^{6} \mathrm{Li}_{\text {g.s. }}$, produced via $n$-stripping of ${ }^{7} \mathrm{Li}[19,27$, since the events corresponding to ${ }^{6} \mathrm{Li}$ could not be separated from those of ${ }^{7} \mathrm{Li}$ in the spectra. However, the $\mathrm{Z}=3$ band predominantly consists of elastic and inelastic events. So, for nomenclature purpose, here we refer the $\mathrm{Z}=3$ band as partial quasielastic scattering band, which represents primarily the sum of elastic and inelastic events. A one-dimensional projection of the $\mathrm{Z}=3$ band was observed to show a quasielastic peak with FWHM of $\approx 500 \mathrm{keV}$ at energy, $\mathrm{E}_{l a b}=29 \mathrm{MeV}$. 


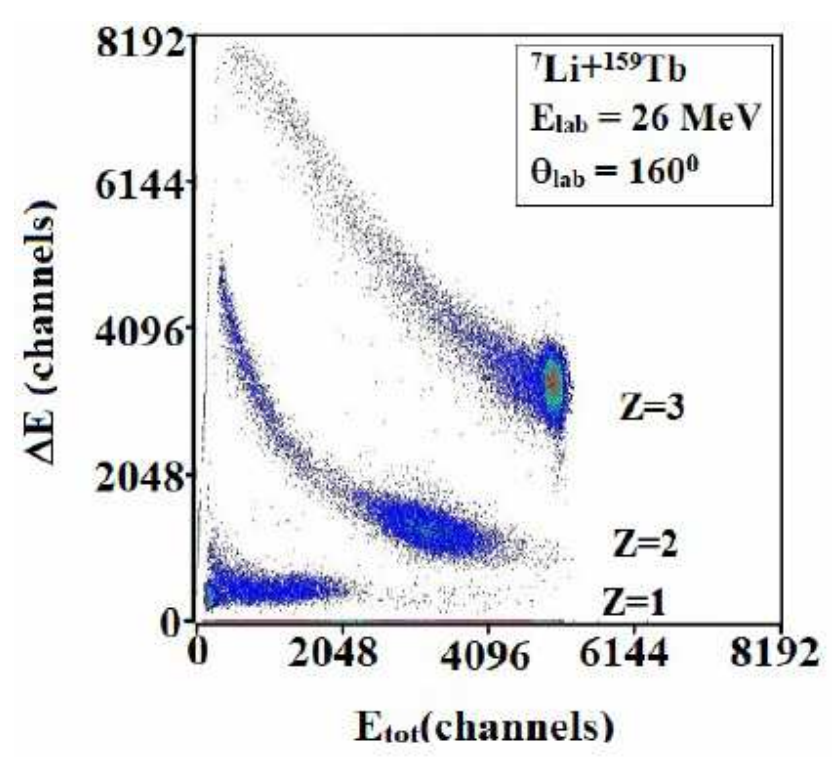

FIG. 1: (Color online) Typical $\Delta \mathrm{E}^{-\mathrm{E}_{t o t}}$ spectrum for the ${ }^{7} \mathrm{Li}+{ }^{159} \mathrm{~Tb}$ reaction at $\mathrm{E}_{l a b}=26 \mathrm{MeV}$ and $\theta_{l a b}=+160^{\circ}$

The $\mathrm{Z}=2$ band corresponds to various events producing $\alpha$-particles in the reaction. A one-dimensional projection of the $\mathrm{Z}=2$ band shows a broad $\alpha$-peak. The contribution of the $\alpha$-particles, emitted mostly at energies corresponding to the beam velocity primarily originate from breakup related processes, like no-capture breakup (NCBU) and ICF. The broad $\alpha$-peak can also have contributions from the transfer of a single nucleon and/ or a cluster of nucleons followed by the breakup process, and thereby resulting in $\alpha$-particles. The processes that might contribute to the $\alpha$-particle cross-sections for the ${ }^{7} \mathrm{Li}+{ }^{159} \mathrm{~Tb}$ reaction are:

(1) NCBU: Breakup of ${ }^{7} \mathrm{Li}$ (B.U threshold for $\alpha+t$ is 2.47 $\mathrm{MeV}$ ) into $\alpha$ and $t$, either direct or sequential or both, where both the fragments escape without any of them being captured by the target

(2) Triton-ICF: $t$ captured by the target following break up of ${ }^{7} \mathrm{Li}$ into $\alpha$ and $t$ or a one step $t$-transfer to the target

(3) Neutron stripping: Single $n$-stripping from ${ }^{7} \mathrm{Li}$ will produce ${ }^{6} \mathrm{Li}$ which may break into $\alpha$ and $d$, if excited above its breakup threshold $1.47 \mathrm{MeV}$

(4) Deuteron stripping: $d$-stripping from ${ }^{7} \mathrm{Li}$ will produce unbound ${ }^{5} \mathrm{He}$ that decays to $\alpha$ and a neutron

(5) Proton stripping: $p$-stripping from ${ }^{7} \mathrm{Li}$ will produce unbound ${ }^{6} \mathrm{He}$ which then decays to $\alpha$ and two neutrons

(6) Proton pickup: p-pickup by ${ }^{7} \mathrm{Li}$ will lead to ${ }^{8} \mathrm{Be}$ which immediately decays to two $\alpha$-particles. Since this is an inclusive measurement, each ${ }^{8} \mathrm{Be}$ will contribute two $\alpha$ particles to the total $\alpha$-yield. But, the contribution of $\alpha$-particles from the $p$-pickup channel may be expected to be very small in comparison to the total contribution of $\alpha$-particles from other processes, like ICF $\left(t+{ }^{159} \mathrm{~Tb}\right)[27$. Hence, the extra $\alpha$-particle contribution arising from the double counting of $\alpha$-particles may be neglected in comparison to the total $\alpha$-particle contribution for the reaction.

The $\mathrm{Z}=1$ band in the figure shows a fall-back feature because the stop detectors were not thick enough to stop the $\mathrm{Z}=1$ particles. So the events corresponding to $\mathrm{Z}=1$ could not be used in the analysis.

The ratio of quasielastic to Rutherford cross-sections is given by the expression,

$$
\begin{gathered}
\frac{d \sigma_{q e l}}{d \sigma_{R u t h}}\left(E, \theta_{t e l}\right)=\left\lfloor\frac{N_{q e l}\left(E, \theta_{t e l}\right)}{N_{m}\left(E, \theta_{m}\right)}\right\rfloor X \\
\left\lfloor\frac{\left(d \sigma_{R u t h} / d \Omega\right)\left(E, \theta_{m}\right)}{\left(d \sigma_{\text {Ruth }} / d \Omega\right)\left(E, \theta_{t e l}\right)}\right\rfloor\left(\frac{\Delta \Omega_{m}}{\Delta \Omega_{t e l}}\right)
\end{gathered}
$$

where,

$\mathrm{N}_{q e l}\left(\mathrm{~N}_{m}\right)$ is the average yield in telescope (monitor) detector,

$\frac{d \sigma_{R u t h}}{d \Omega}\left(E, \theta_{m}\left(\theta_{t e l}\right)\right)$ is the calculated Rutherford scattering cross-section at the corresponding bombarding energy $\mathrm{E}$, at monitor angle $\theta_{m}$ (telescope angle $\theta_{t e l}$ ), and

$\left(\frac{\Delta \Omega_{m}}{\Delta \Omega_{t e l}}\right)$ is the solid angle ratio of monitor to telescope detectors.

The $\frac{\Delta \Omega_{m}}{\Delta \Omega_{t e l}}$ ratio for each of the four telescope angles was determined from the measurements at the lowest bombarding energies of 17,18 and $19 \mathrm{MeV}$, where the elastic scattering is purely Rutherford.

The "partial" quasielastic counts $N_{q e l}\left(E, \theta_{t e l}\right)$ at each bombarding energy were obtained from the sum of elastic and inelastic counts in the $\mathrm{Z}=3$ band in Fig. 1 1 . As the measurements were done at angles close to $180^{\circ}$, centrifugal correction was incorporated to obtain the effective c.m. energies $\left(E_{\text {eff }}\right)$. The results of the quasielastic events at $\pm 170^{\circ}$ and $\pm 160^{\circ}$ were converted to those for $180^{\circ}$ by mapping to $E_{\text {eff }}$ using the relation [4],

$$
E_{e f f}=\frac{2 E_{c . m .}}{1+\operatorname{cosec} \frac{\theta_{c . m .}}{2}}
$$

To check the consistency of the data, the quasielastic excitation functions and barrier distributions were extracted using the data taken at $\pm 170^{\circ}$ and $\pm 160^{\circ}$, and after appropriate centrifugal correction they were found to agree fairly well each other. The good agreement between the measurements at different angles gave us confidence in our data.

The "partial" quasielastic scattering excitation function determined from the $\mathrm{Z}=3$ events and the corresponding quasielastic barrier distribution, $\mathrm{D}_{q e l}$, extracted using Eq. (1) are shown by the solid circles (•) in Figs. 2(a) and 2(b), respectively. The $\mathrm{E}_{c . m}$. energies in the figures are essentially $E_{\text {eff }}$ energies. The quasielastic cross sections in Fig. 2(a) were obtained by averaging the cross sections for the two telescopes at $\pm 170^{\circ}$, after appropriate centrifugal corrections. The barrier distribution shown by the solid circles $(\bullet)$ in Fig. 2(b) was derived from the "partial" quasielastic excitation function, instead of the "total" quasielastic cross sections which would include all 
relevant reaction channels and not only elastic and inelastic events. So the derived barrier distribution, shown in Fig. 2(b) by the solid circles (•), does not correspond to the fusion barrier distribution, but rather reflect the reaction threshold distribution [9]. The quasielastic scattering cross sections and the corresponding barrier distribution, determined from the sum of $\mathrm{Z}=3$ (elastic + inelastic) and $\mathrm{Z}=2(\alpha)$ events, are also shown in Figs. 2(a) and 2 (b) by the solid triangles $(\boldsymbol{\Delta})$. It is observed that the inclusion of $\alpha$-particles in the definition of quasielastic events, shifts the centroid of the barrier distribution higher by $\approx 800 \mathrm{keV}$ than the distribution corresponding to only $\mathrm{Z}=3$ events. A similar observation has also been reported for the system ${ }^{7} \mathrm{Li}+{ }^{197} \mathrm{Au}[20]$.

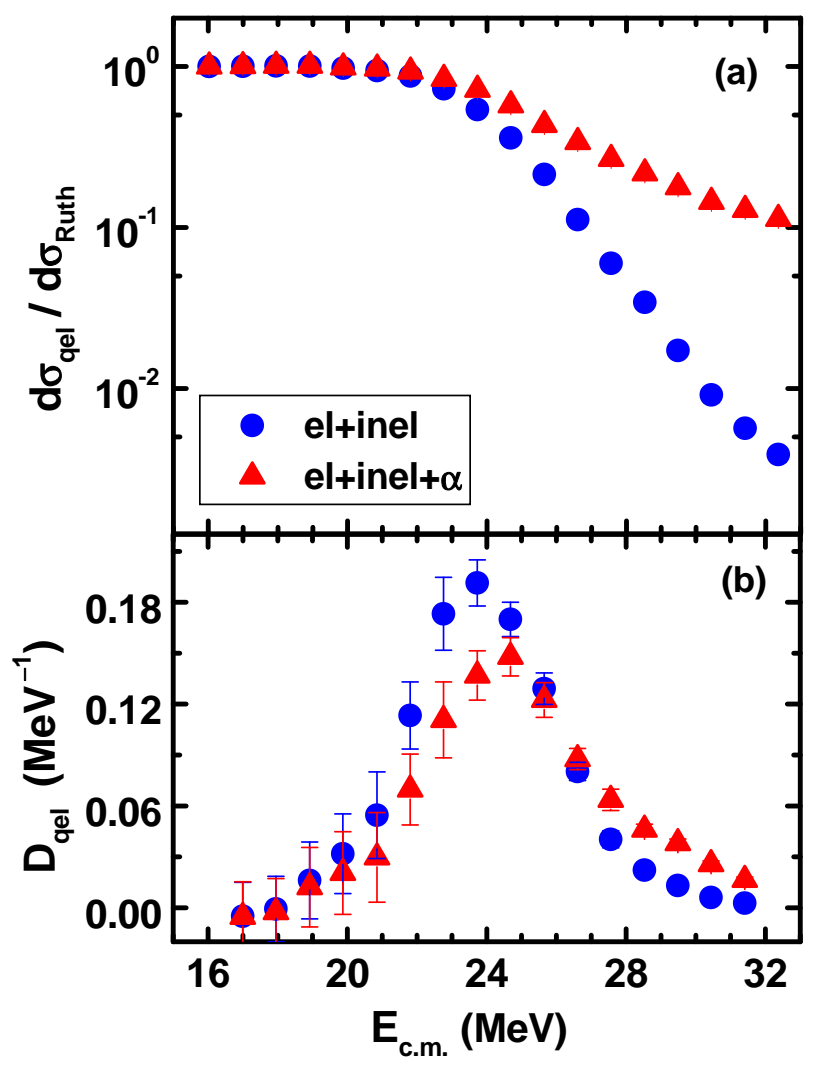

FIG. 2: (Color online) Comparison of a) quasielastic excitation function and b) quasielastic barrier distribution for ${ }^{7} \mathrm{Li}+{ }^{159} \mathrm{~Tb}$, excluding $(\bullet)$ and including $(\boldsymbol{\Delta})$ the $\alpha$ particles produced in the reaction.

\section{COUPLED CHANNEL CALCULATIONS}

In this section, our primary focus is to investigate the effects of couplings between different reaction channels at near-barrier energies in the CRC framework, and not the relation between $D_{f u s}$ and $D_{q e l}$. So, here we considered the "partial" quasielastic scattering cross sections derived only from the $\mathrm{Z}=3$ events. This is because the $\mathrm{Z}=1$ particles could not be stopped in the detectors and the different reaction channels contributing to the $\mathrm{Z}=2$ events, discussed above, could not be disentangled in the present inclusive measurement. The coupled channel calculations were carried out to analyze the measured "partial" quasielastic scattering excitation function and barrier distribution for ${ }^{7} \mathrm{Li}+{ }^{159} \mathrm{~Tb}$, employing the code FRESCO (version FRES 2.9) [28].

The primary input for the CRC calculations is the entrance channel optical potential which consists of Coulomb potential plus the bare nuclear potential. The bare nuclear potential parameters for a system are derived from an optical model analysis of a set of measured elastic scattering differential cross sections for the system. But measuring pure elastic scattering cross sections for the system ${ }^{7} \mathrm{Li}+{ }^{159} \mathrm{~Tb}$ is experimentally very difficult because of the very closely spaced low-lying excited energy levels of ${ }^{159} \mathrm{~Tb}$. The only elastic scattering angular distribution data available in the literatute for ${ }^{7} \mathrm{Li}+{ }^{159} \mathrm{~Tb}$ are those of Ref. 29]. But these data have contributions from the low-lying excited states of ${ }^{159} \mathrm{~Tb}$. So, bare nuclear potential parameters could not be obtained for the system ${ }^{7} \mathrm{Li}+{ }^{159} \mathrm{~Tb}$.

The elastic scattering angular distribution data [29] were therefore re-analyzed in the present work, in the CDCC framework, to obtain a set of properly adjusted cluster folding potentials for $\alpha+{ }^{159} \mathrm{~Tb}$ and $t+{ }^{159} \mathrm{~Tb}$, where the ${ }^{7} \mathrm{Li}$ nucleus was considered to have a $\alpha+t$ cluster structure with B.U. threshold of $2.47 \mathrm{MeV}$. The CDCC calculations were done using the code FRESCO. The continuum of ${ }^{7} \mathrm{Li}$ above the B.U. threshold of 2.47 $\mathrm{MeV}$ consists of non-resonant and resonant states. It has been observed 30] that in the CDCC calculations for elastic scattering angular distribution of the relatively more weakly bound ${ }^{6} \mathrm{Li}$-induced reactions, couplings due to resonant states are more dominant compared to the non-resonant ones. So, for the ease of calculations, for the $\mathrm{CDCC}$ model space of ${ }^{7} \mathrm{Li}$ we considered only the low-lying non-resonant continuum states up to an excitation energy of $4.4 \mathrm{MeV}$ of ${ }^{7} \mathrm{Li}$ and the two resonant states $7 / 2^{-}$and $5 / 2^{-}$at 4.63 and $6.68 \mathrm{MeV}$, respectively. Continuum states with angular momentum $l=0$, 1, 2 and 3 were considered. The non-resonant continuum was discretized into momentum bins of width $\Delta k=0.2$ $\mathrm{fm}^{-1}$, only up to $k_{\max }=0.4 \mathrm{fm}^{-1}$. The binning of the continuum with $l=3$ was suitably done so as to include the two resonant states, $7 / 2^{-}$and $5 / 2^{-}$with average excitation energies of 2.16 and $4.21 \mathrm{MeV}$ relative to the B.U. threshold of ${ }^{7} \mathrm{Li}$ and widths of $0.2 \mathrm{MeV}$ and $3.0 \mathrm{MeV}$, respectively [31]. The widths taken were sufficient enough to accommodate the main strength of the resonances. To calculate the bin wavefunctions, the binding potential between $\alpha$ and $t$ for bound and resonant states of ${ }^{7} \mathrm{Li}$ projectile were taken from Ref. 31]. The wavefunction of the projectile-target relative motion was expanded in partial waves up to $\mathrm{J}_{\max }=150$ and it was integrated numerically up to $140 \mathrm{fm}$, in steps of $0.05 \mathrm{fm}$. In addition to the continuum of ${ }^{7} \mathrm{Li}$, the bound excited state of ${ }^{7} \mathrm{Li}$, having spin $1 / 2^{-}$and $\mathrm{E}_{e x}=0.477 \mathrm{MeV}$, with reduced tran- 
TABLE I: Reduced transition probabilities B(E2) [32] used in the coupled channel analysis for the calculations of the Coulomb matrix elements and nuclear deformation lengths for inelastic transitions in ${ }^{159} \mathrm{~Tb}$

\begin{tabular}{|c|c|}
\hline $\begin{array}{l}\text { Transition } \\
\left(J_{i} \rightarrow J_{f}\right) \\
\end{array}$ & $\begin{array}{c}\mathrm{B}\left(\mathrm{E} 2 ; J_{i} \rightarrow J_{f}\right) \\
\left(\mathrm{e}^{2} \mathrm{~b}^{2}\right)\end{array}$ \\
\hline$\overline{5 / 2 \rightarrow 3 / 2}$ & $\overline{\overline{1.87}}$ \\
\hline $7 / 2 \rightarrow 5 / 2$ & 1.25 \\
\hline $7 / 2 \rightarrow 3 / 2$ & 0.72 \\
\hline $9 / 2 \rightarrow 7 / 2$ & 0.61 \\
\hline $9 / 2 \rightarrow 5 / 2$ & 1.13 \\
\hline $11 / 2 \rightarrow 9 / 2$ & 0.56 \\
\hline $11 / 2 \rightarrow 7 / 2$ & 1.47 \\
\hline $13 / 2 \rightarrow 11 / 2$ & 0.32 \\
\hline $13 / 2 \rightarrow 9 / 2$ & 1.74 \\
\hline
\end{tabular}

sition probability $B(E 2 \uparrow)=8.3 e^{2} \mathrm{fm}^{4}[20]$ was included in the coupling scheme. Also, the two low-lying excited states, $5 / 2^{+}$state at $0.058 \mathrm{MeV}$ and $7 / 2^{+}$state at 0.137 $\mathrm{MeV}$, of ${ }^{159} \mathrm{~Tb}$ were included in the coupling scheme 29$]$. The B(E2) values [32] for the corresponding transitions in ${ }^{159} \mathrm{~Tb}$, used in the calculations are listed in Table I. The Coulomb reduced matrix elements and the nuclear deformation lengths for the coupled channel calculations were derived from the $B(E 2)$ values, assuming rotational model for ${ }^{159} \mathrm{~Tb}$. Adjusting the cluster folded potentials of ${ }^{7} \mathrm{Li}+{ }^{159} \mathrm{~Tb}$, and fixing them at depth $V_{0}=23.9 \mathrm{MeV}$, radius parameter, $r_{0}=1.2 \mathrm{fm}$ and diffuseness, $a=0.5 \mathrm{fm}$ for $\alpha+{ }^{159} \mathrm{~Tb}$ and at $V_{0}=29.9 \mathrm{MeV}, r_{0}=1.2 \mathrm{fm}$ and $a=0.5$ $\mathrm{fm}$ for $t+{ }^{159} \mathrm{~Tb}$, gave a reasonable description of the elastic scattering angular distribution data [29] at incident energy $\mathrm{E}_{l a b}=35 \mathrm{MeV}$. The imaginary parts of the potentials were taken to be of Woods-Saxon form, with $W_{0}=50 \mathrm{MeV}, r_{0}=1.0 \mathrm{fm}$ and $a=0.4 \mathrm{fm}$. All reorientation couplings have been considered in the calculations. Figure 3 compares the angular distribution data of Ref. [29], at $\mathrm{E}_{l a b}=35 \mathrm{MeV}$, with the CDCC calculated cross sections. The solid line in the figure shows the calculated cross sections in the above coupling scheme, where coupling to both resonant and non-resonant parts of the continuum of ${ }^{7} \mathrm{Li}$ was included. Fairly good agreement is observed between the calculated and measured angular distribution cross sections. To see the importance of the effect of coupling to non-resonant part of the continuum of ${ }^{7} \mathrm{Li}$ on the elastic scattering angular distribution, the same calculations were repeated excluding the nonresonant continuum states of ${ }^{7} \mathrm{Li}$ in the above coupling scheme. The calculated elastic scattering angular distribution at $\mathrm{E}_{l a b}=35 \mathrm{MeV}$ thereby obtained are shown by the dashed line in Fig. 3, The very good agreement between the two calculations confirms that for elastic scattering angular distribution of ${ }^{7} \mathrm{Li}+{ }^{159} \mathrm{~Tb}$, couplings due to resonant states of ${ }^{7} \mathrm{Li}$ are more dominant compared to the non-resonant ones.

Now that it is established that the effect of the resonant states of ${ }^{7} \mathrm{Li}$ on the elastic scattering angular distri-

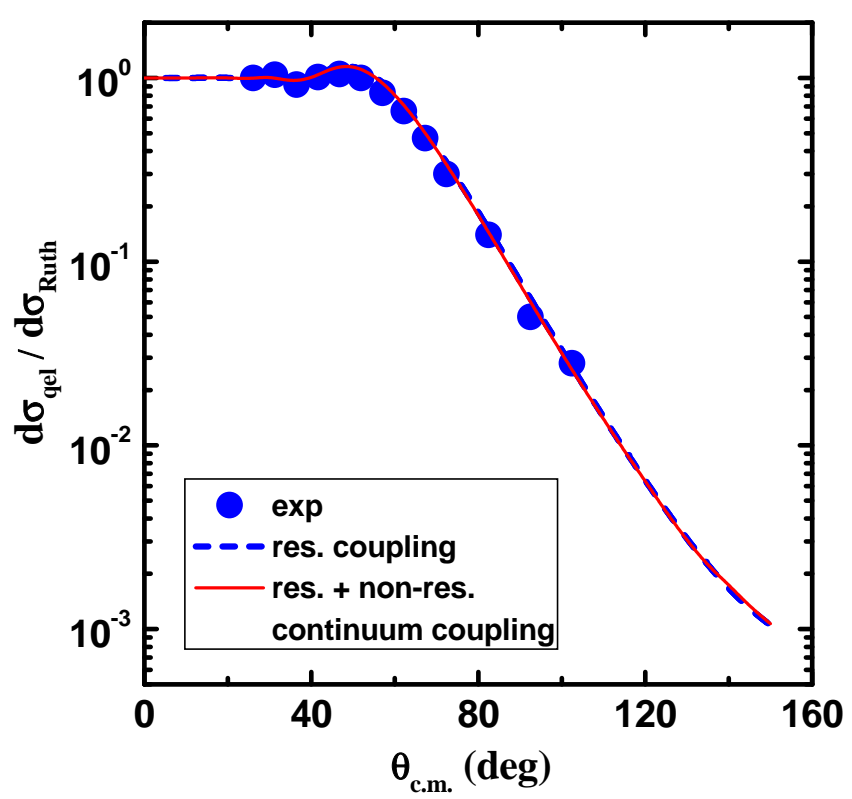

FIG. 3: (Color online) Elastic scattering angular distribution for the ${ }^{7} \mathrm{Li}+{ }^{159} \mathrm{~Tb}$ system at $\mathrm{E}_{l a b}=35 \mathrm{MeV}$ [29]. The solid and dashed lines represent the CDCC calculations with and without coupling to non-resonant continuum states of ${ }^{7} \mathrm{Li}$. Coupling to resonant states in the continuum of ${ }^{7} \mathrm{Li}$ were included in both the calculations.

bution of ${ }^{7} \mathrm{Li}+{ }^{159} \mathrm{~Tb}$ are dominant compared to the nonresonant continuum states, in the present re-analysis of the elastic scattering angular distribution data [29] coupling to non-resonant continuum states was not considered in further calculations where continuum coupling is used. Hereafter, for continuum coupling, only the two resonant states, $7 / 2^{-}$at $4.63 \mathrm{MeV}$ and $5 / 2^{-}$at $6.68 \mathrm{MeV}$, of ${ }^{7} \mathrm{Li}$ were considered in the CDCC coupling scheme. The CDCC calculations with the above coupling scheme, excluding the non-resonant continuum of ${ }^{7} \mathrm{Li}$, and using the cluster folded potentials for $\alpha+{ }^{159} \mathrm{~Tb}$ and $t+{ }^{159} \mathrm{~Tb}$, as obtained above, the elastic scattering angular distributions for ${ }^{7} \mathrm{Li}+{ }^{159} \mathrm{~Tb}$ were calculated at $\mathrm{E}_{l a b}=26,28$, 30, 35 and $44 \mathrm{MeV}$. Figure 4 compares the angular distribution data of Ref. [29] with the theoretical cross sections at different bombarding energies. The solid lines in the figure show the calculated cross sections. Fairly good agreement can be seen between the calculated and experimental elastic scattering angular distribution cross sections.

Having reproduced the elastic scattering angular distribution data reasonably well, the above coupling scheme was used to calculate the large back-angle quasielastic scattering excitation function for ${ }^{7} \mathrm{Li}+{ }^{159} \mathrm{~Tb}$. The calculations were performed with different coupling conditions:

(i) No coupling to the continuum of ${ }^{7} \mathrm{Li}$ was considered. Only inelastic coupling to low-lying excited states of ${ }^{159} \mathrm{~Tb}$ and bound state of ${ }^{7} \mathrm{Li}$ at $0.477 \mathrm{MeV}$ were included. 


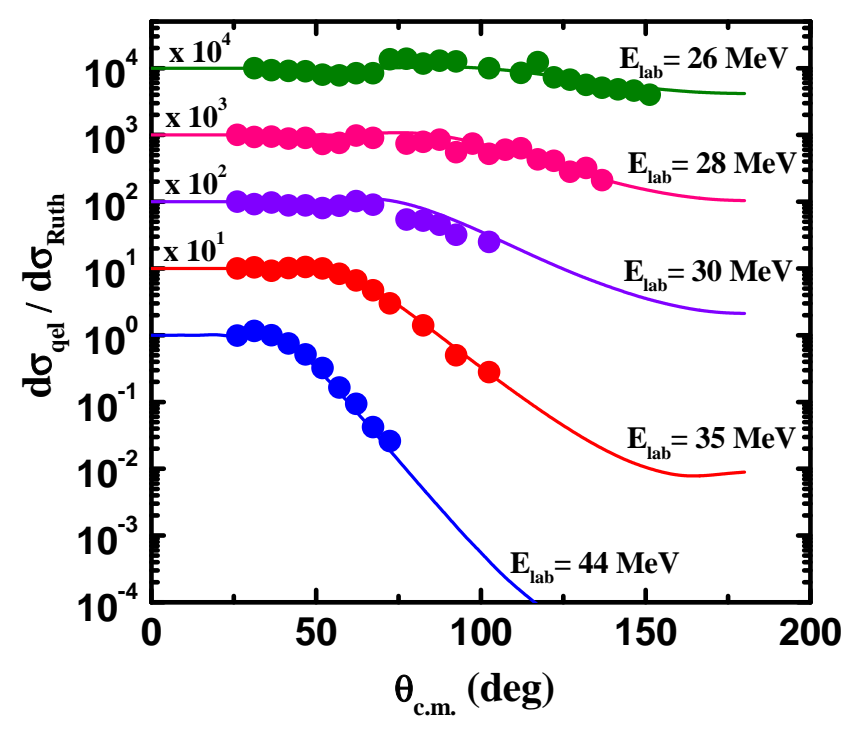

FIG. 4: (Color online) Elastic scattering angular distributions for the ${ }^{7} \mathrm{Li}+{ }^{159} \mathrm{~Tb}$ system [29], at different bombarding energies. The solid lines represent the CDCC calculations.

(ii) Only couplings to two resonant states, $7 / 2^{-}$and $5 / 2^{-}$ at 4.63 and $6.68 \mathrm{MeV}$, in the continuum of ${ }^{7} \mathrm{Li}$ were included. In this scheme, couplings to neither target excited states, nor bound excited state of ${ }^{7} \mathrm{Li}$ were considered.

(iii) Subsequently, calculations with couplings only to the above two resonant states in ${ }^{7} \mathrm{Li}$ continuum, the bound excited state of ${ }^{7} \mathrm{Li}$ and the low-lying excited states of ${ }^{159} \mathrm{~Tb}$ were done.

(iv) Finally, full CDCC calculations with couplings to both resonant and non-resonant states of ${ }^{7} \mathrm{Li}$ continuum, along with couplings to the bound excited state of ${ }^{7} \mathrm{Li}$ and the low-lying excited states of ${ }^{159} \mathrm{~Tb}$ were performed. The results of these calculations are discussed below.

The calculations were first performed with coupling condition (i). The dot-dashed lines in Figs. 5 and 6 represent the quasielastic scattering excitation function and the corresponding barrier distribution, calculated with inelastic coupling only up to the first excited state of ${ }^{159} \mathrm{~Tb}$ at $5 / 2^{+}$. The dotted lines in the figures are the no-coupling calculations. It has already been mentioned in Section II that the quasielastic peak for the $\mathrm{Z}=3$ band had a FWHM of $\approx 500 \mathrm{keV}$ at $\mathrm{E}_{l a b}=29 \mathrm{MeV}$. So, calculations including inelastic excitation of ${ }^{159} \mathrm{~Tb}$ up to $13 / 2^{+}$ at $0.510 \mathrm{MeV}$ in the coupling scheme, were done. The dashed line in the Fig. [5 shows the quasielastic excitation function, thereby calculated. The corresponding barrier distribution is plotted in Fig. 6. No significant change is observed either in excitation function or barrier distribution, if inelastic excited states of ${ }^{159} \mathrm{~Tb}$ beyond $5 / 2^{+}$are included in the coupling scheme. This indicates that the

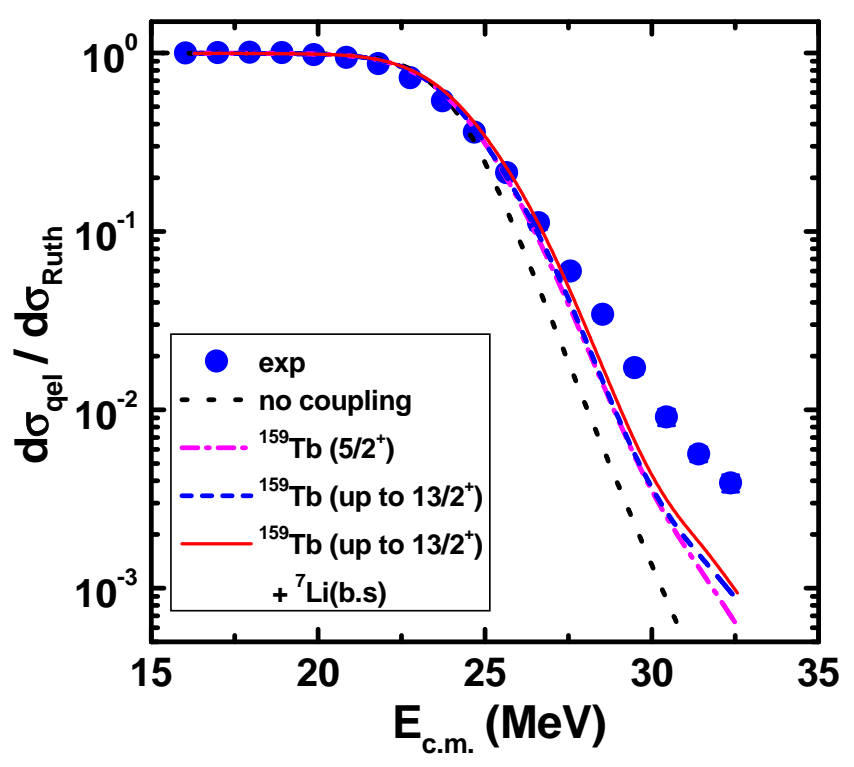

FIG. 5: (Color online) Comparison of the measured partial quasielastic excitation function for the ${ }^{7} \mathrm{Li}+{ }^{159} \mathrm{~Tb}$ system compared with the coupled channel calculations with different inelastic coupling conditions. See text for details.

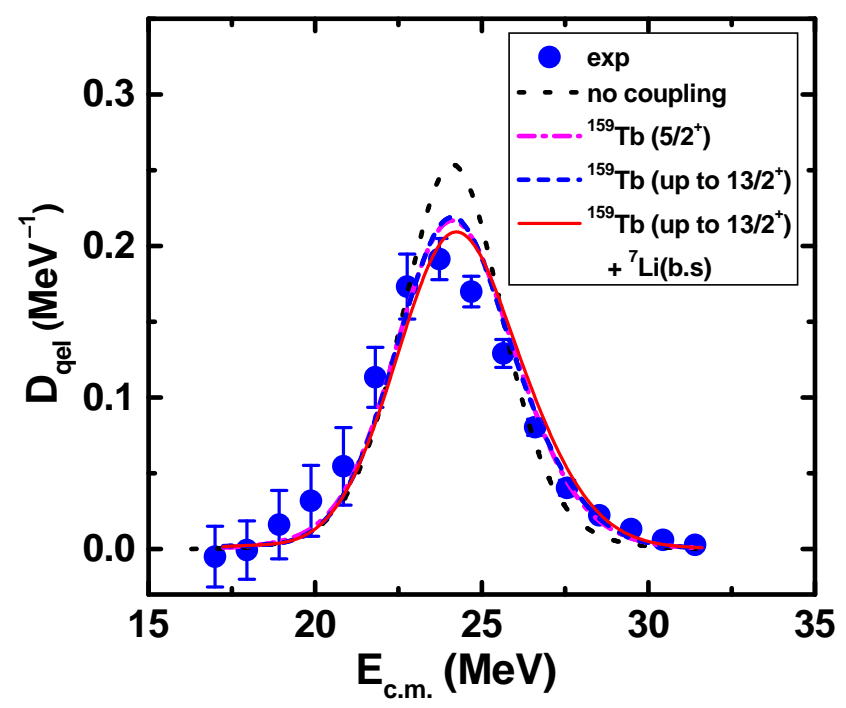

FIG. 6: (Color online) Comparison of the partial quasielastic barrier distribution for the ${ }^{7} \mathrm{Li}+{ }^{159} \mathrm{~Tb}$ system compared with coupled channel predictions with different inelastic coupling conditions. See text for details.

$5 / 2^{+}$state of ${ }^{159} \mathrm{~Tb}$ is a strong contributor to the target inelastic coupling. Figures 5 and 6 show that inclusion of only inelastic excited states of ${ }^{159} \mathrm{~Tb}$ in the coupling scheme fails to reproduce the quasielastic scattering excitation function and barrier distribution for ${ }^{7} \mathrm{Li}+{ }^{159} \mathrm{~Tb}$. So, projectile excitation was then considered, in addition to the target excitation, by including the bound excited state of ${ }^{7} \mathrm{Li}$ at $0.477 \mathrm{MeV}$ in the coupling scheme. 
For comparison with experimental results, the quasielastic scattering cross sections were determined by adding the calculated elastic cross sections to the cross sections of the inelastic states up to $13 / 2^{+}$of ${ }^{159} \mathrm{~Tb}$ and the bound excited state of ${ }^{7} \mathrm{Li}$, and are shown by the solid line in Fig. 5 The corresponding barrier distribution is shown by the solid line in Fig. 6. The calculations are still seen to underestimate the measured quasielastic excitation function at higher energies and also fail to reproduce the experimental barrier distribution.

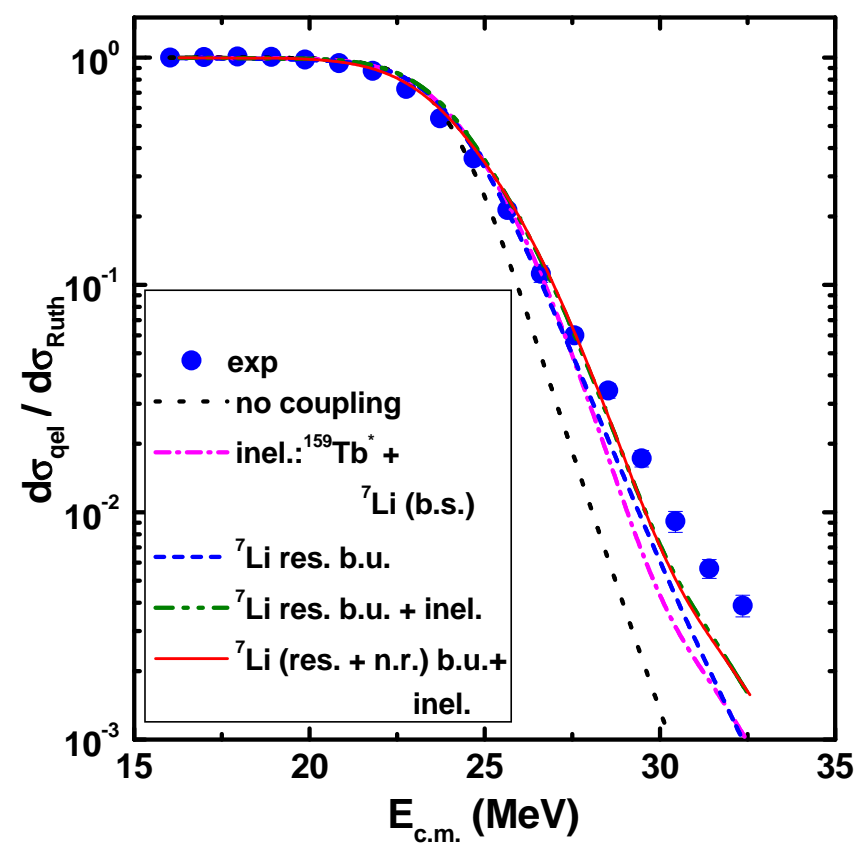

FIG. 7: (Color online) Effect of full coupling to the ${ }^{7} \mathrm{Li}$ continuum in addition to the inelastic coupling to ${ }^{7} \mathrm{Li}$ and ${ }^{159} \mathrm{~Tb}$ bound excited states on the quasielastic scattering excitation function for the ${ }^{7} \mathrm{Li}+{ }^{159} \mathrm{~Tb}$ system. See text for details.

The CDCC calculations were then repeated with coupling condition (ii), i.e., couplings only to the two resonant states, $7 / 2^{-}$and $5 / 2^{-}$at 4.63 and $6.68 \mathrm{MeV}$, respectively, in the continuum of ${ }^{7} \mathrm{Li}$. The resulting quasielastic excitation function and barrier distribution are shown by the dashed lines in Figs. 7 and [8, respectively. Subsequently, the calculations were repeated with coupling condition (iii), i.e., couplings to the above two resonant states in the continuum of ${ }^{7} \mathrm{Li}$ and also projectile and target inelastic couplings of case (i). The results are shown by the dot-dot-dashed lines in the figures. It can be seen that the inclusion of coupling to the resonant states of ${ }^{7} \mathrm{Li}$ continuum, in addition to the inelastic couplings to the bound excited state of ${ }^{7} \mathrm{Li}$ and the low-lying excited states of ${ }^{159} \mathrm{~Tb}$ better reproduces the quasielastic scattering excitation function except at higher energies, but not much change is observed in the barrier distribution. It is observed that the effect of couplings to the channels included in coupling scheme (iii) essentially reduces the height of the quasielastic barrier distribution

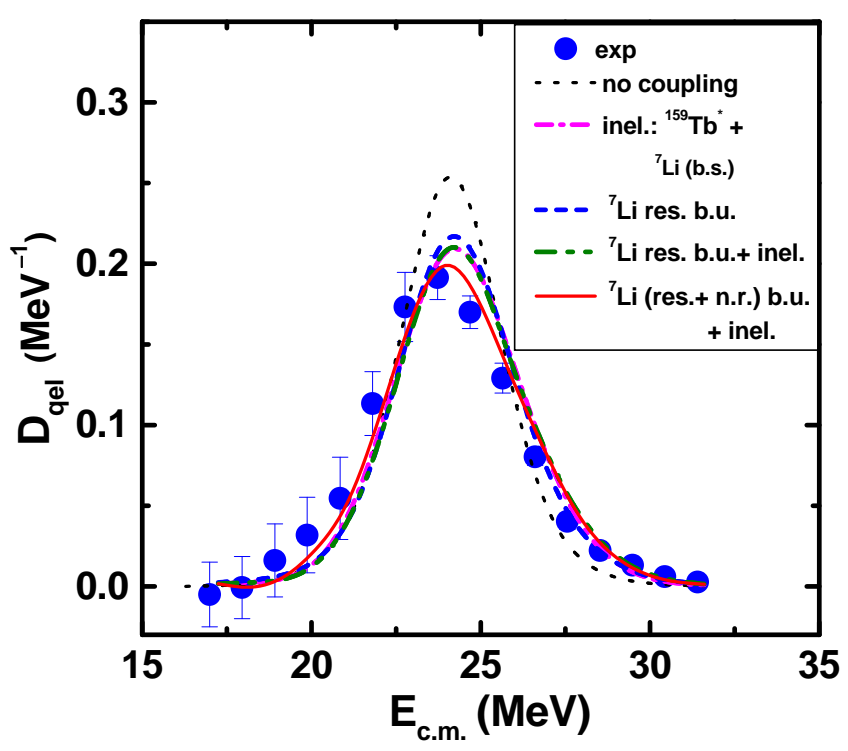

FIG. 8: (Color online) Effect of full coupling to the ${ }^{7} \mathrm{Li}$ continuum in addition to the inelastic couping to ${ }^{7} \mathrm{Li}$ and ${ }^{159} \mathrm{~Tb}$ bound excited states on the quasielastic barrier distribution for the ${ }^{7} \mathrm{Li}+{ }^{159} \mathrm{~Tb}$ system. See text for details.

and also broadens the distribution, as compared to the no-coupling calculations.

Finally, full CDCC calculations were performed with coupling condition (iv), i.e., couplings to both resonant and non-resonant states in continuum of ${ }^{7} \mathrm{Li}$ along with the inelastic couplings to ${ }^{7} \mathrm{Li}$ bound excited state $\left(1 / 2_{-}\right.$, $0.477 \mathrm{MeV}$ ) and ${ }^{159} \mathrm{~Tb}$ low-lying excited states of case (i). The CDCC model space of ${ }^{7} \mathrm{Li}$ was discretized into small bins of width $\Delta k=0.2 \mathrm{fm}^{-1}$ up to $k_{\max }=0.8 \mathrm{fm}^{-1}$. The resonant states were treated appropriately to avoid double counting. Continuum states with angular momentum $l=0,1,2$ and 3 were considered. For lower bombarding energies, the convergence is reached by decreasing the upper limit of the excitation energy. Other details of the scheme are discussed above in the CDCC calculations for elastic scattering angular distribution. The results of the calculations are shown by the solid lines in Figs. 7 and 8. The inclusion of non-resonant part of the ${ }^{7} \mathrm{Li}$ continuum in the calculations significantly affects the height and location of the centroid of the quasielastic barrier distribution, though no considerable change is observed in the quasielastic excitation function. The height of the barrier distribution is now almost similar to the experimental barrier distribution, and the centroid of the distribution has also shifted considerably towards the experimental barrier distribution.

It needs to be pointed out that the effects due to couplings to transfer and transfer induced breakup channels have not been explored here. Inclusion of these couplings may better reproduce the quasielastic excitation function at the higher energies. The small shift observed between the centroids of the experimental 
and theoretical barrier distributions could be due to the reaction channels, especially transfer induced breakup, not included in the calculations.

\section{COMPARISON OF $D_{q e l}$ AND $D_{f u s}$}

A comparison of the $D_{q e l}$, including and excluding the $\alpha$ particle contribution, with the $D_{\text {fus }}$ may shed some light on the importance of the $\alpha$ particle contribution in defining the quasielastic scattering events for weakly bound systems.

To compare $D_{q e l}$ with $D_{f u s}$, an attempt was made to extract the $D_{\text {fus }}$ from the measured complete fusion (CF) excitation function for ${ }^{7} \mathrm{Li}+{ }^{159} \mathrm{~Tb}[24,33$. Unfortunately, $D_{\text {fus }}$ could not be extracted from the reported fusion cross sections [24, 33], because only a few data points were available for differentiation. Therefore, a rough comparison of the experimental $D_{q e l}$ was made with the theoretical $D_{f u s}$, extracted from the calculated fusion cross sections which reproduced the measured $\mathrm{CF}$ cross sections [24, 33].

Following Ref. 24], the fusion cross sections were calculated using the coupled channels code, CCFULL 34 with Akyuz Winther potential and all other parameters as mentioned in the reference. In addition to the coupling scheme used in Ref. [24], in the present work coupling to the bound excited state of the projectile ${ }^{7} \mathrm{Li}$, having spin $1 / 2^{-}$and $E_{e x}=0.477 \mathrm{MeV}$ was also included using the rotational scheme 20]. Figure 9]compares the experimental CF cross sections [24, 33] with the calculated fusion cross sections. The dotted curve shows the no coupling calculations [24]. The dot-dot-dashed line (CC1) shows the coupled channels calculation considering rotational coupling to six excited states of ${ }^{159} \mathrm{~Tb}[24]$. The dashed line (CC2) shows the coupled channels calculations including rotational coupling to six excited states of ${ }^{159} \mathrm{~Tb}$ and also rotational coupling to the bound excited state of ${ }^{7} \mathrm{Li}$. The measured complete fusion cross sections for ${ }^{7} \mathrm{Li}+{ }^{159} \mathrm{~Tb}$ at above barrier energies are known to be suppressed by a factor of 0.74 compared to the fusion cross sections obtained from coupled channel calculations 24]. The solid line in Fig. 9 shows the CC2 cross sections, after being scaled by a factor of 0.74 , and will be referred hereafter as the calculated CF excitation function. The fusion barrier distributions, $D_{\text {fus }}$ corresponding to calculated CC2 and CF cross sections were then obtained using equation (1).

To compare $D_{q e l}$ with $D_{f u s}$, the $D_{f u s}$ values thus obtained were normalized by $1 / \pi R_{b}^{2}$, where the barrier radius $R_{b}$ was taken from Ref. [24]. Figure 10] shows a comparison of the experimental $D_{q e l}$, including and excluding the contribution of the $\alpha$-particles, with the theoretical $D_{f u s}$ (normalized). The dotted and the dashed lines represent the $D_{\text {fus }}$ extracted from the calculated fusion cross sections without and with coupling (CC2),

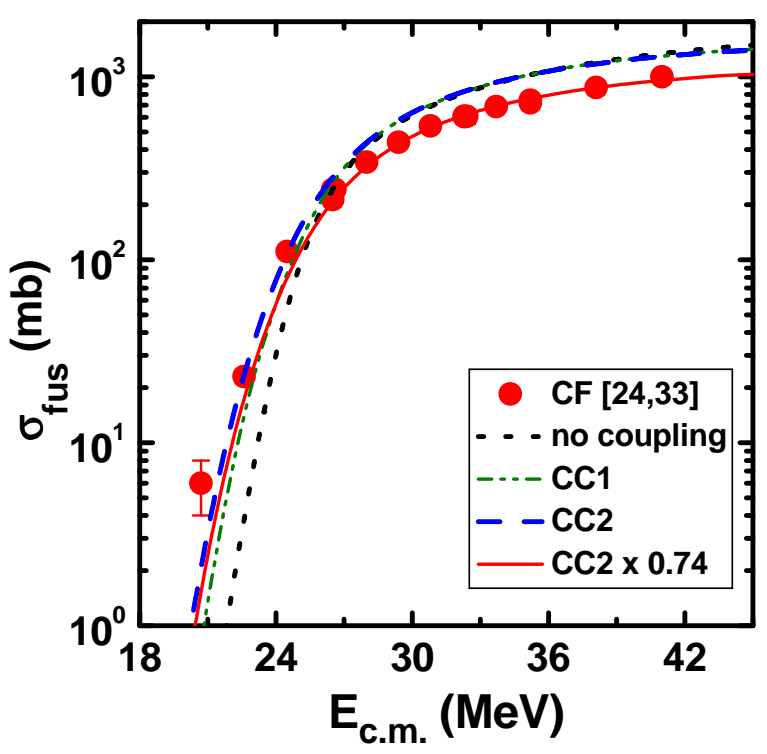

FIG. 9: (Color online) Complete fusion cross sections for the ${ }^{7} \mathrm{Li}+{ }^{159} \mathrm{~Tb}$ system 24]. The dotted curve shows the no coupling calculations. The dot-dash-dashed (CC1) and dashed (CC2)lines are the coupled channels calculations performed with the code CCFULL 34]. The solid line shows the coupled channels calculations (CC2) scaled by the factor 0.74 .

respectively. The solid line shows the results obtained when $D_{\text {fus }}$ (normalized) is derived from the calculated $\mathrm{CF}$ cross sections 20]. It can be seen from the figure that the peak of $D_{q e l}$ excluding the contribution of $\alpha$-particles (shown by the symbol •) lies at an energy slightly lower than that of $D_{f u s}$ calculated from the CC2 cross sections and shown by the dashed line. This observation is consistent with those of Refs. [8] and [9].

The experimental $D_{q e l}$ including the contribution of the $\alpha$-particles and shown by the symbol $\boldsymbol{\Delta}$ in the figure is found to agree reasonably well with the calculated CF $D_{\text {fus }}$, except a small mismatch at the higher energies. Similar observation has also been reported for the system ${ }^{7} \mathrm{Li}+{ }^{208} \mathrm{~Pb}$ [8]. This indicates that the agreement of $D_{q e l}$, including the contribution of $\alpha$-particles, and $D_{f u s}$ might be independent of the structure of target nuclei. The similarity of CF barrier distribution with the QEL barrier distribution, including the alpha contribution, for ${ }^{7} \mathrm{Li}-$ induced reactions might be understood in the following way.

For weakly bound systems, quasielastic scattering cross sections $\left(\sigma_{q e l}\right)$, are given by,

$$
\sigma_{q e l}=1-\left(\sigma_{C F}+\sigma_{I C F}\right)
$$

where $\sigma_{C F}$ and $\sigma_{I C F}$ are CF and ICF cross sections, respectively. For ${ }^{7} \mathrm{Li}+{ }^{159} \mathrm{~Tb}$ reaction, $t$-capture process is the dominant ICF contributor with $\alpha$-capture process being relatively less significant [24]; an observation also reported for ${ }^{7} \mathrm{Li}+{ }^{124} \mathrm{Sn}$ 35]. So, for ${ }^{7} \mathrm{Li}$-induced reactions, $\sigma_{I C F} \approx \sigma_{t-\text { capture }}$. 


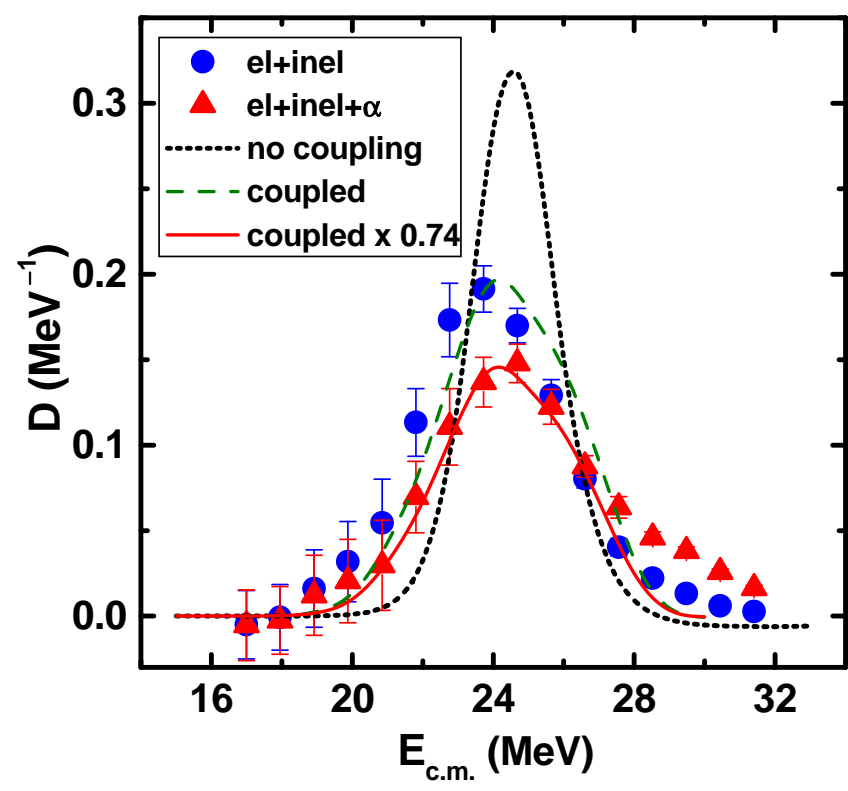

FIG. 10: (Color online) Comparison of the barrier distribution obtained from the quasielastic excitation function with and without inclusion of $\alpha$ particles for the system ${ }^{7} \mathrm{Li}+{ }^{159} \mathrm{~Tb}$. The dotted and the dashed lines represent the $D_{\text {fus }}$ extracted from the coupled channel calculated fusion cross section without and with (CC2) coupling, respectively. The solid line shows the theoretical CF $D_{f u s}$, obtained by scaling the CC2 cross sections by a factor of 0.74 . The theoretical $D_{f u s}$ values have been normalized by the factor $1 /\left(\pi R_{b}^{2}\right)$ to compare with $D_{q e l}$. See text for details.

It has also been reported [36] that for ${ }^{7} \mathrm{Li}$-induced reactions,

$$
\sigma_{t-\text { capture }}=\sigma_{\alpha}-\sigma_{\alpha-C N},
$$

where $\sigma_{\alpha}$ and $\sigma_{\alpha-C N}$ represent the inclusive $\alpha$-yield and the contribution of $\alpha$-particles originating from the decay of the compound nucleus $(\mathrm{CN})$ produced in the $\mathrm{CF}$ process, respectively. But the $\mathrm{CN}$ formed in the fusion of ${ }^{7} \mathrm{Li}$ with ${ }^{159} \mathrm{~Tb}$ and other heavy mass targets decays predominantly by $x n$ evaporation at near-barrier energies [24, 36], and hence $\sigma_{\alpha-C N}$ is expected to be negligible for such systems. Therefore, for ${ }^{7} \mathrm{Li}$-induced reactions with heavy mass targets, $\sigma_{I C F} \approx$ $\sigma_{t-\text { capture }} \approx \sigma_{\alpha}$, which in conjunction with eqn. (5) gives

$$
\sigma_{q e l}+\sigma_{\alpha} \approx 1-\sigma_{C F}
$$

This perhaps explains, why $D_{q e l}$ obtained from the sum of quasielastic and $\alpha$-contributions reasonably agrees with the $\mathrm{CF}$ barrier distribution for ${ }^{7} \mathrm{Li}+{ }^{159} \mathrm{~Tb}$ and ${ }^{7} \mathrm{Li}+{ }^{208} \mathrm{~Pb}[8]$.

However, before reaching any conclusion, one needs to carry out simultaneous measurement of large back-angle quasielastic scattering and fusion excitation functions, and compare the corresponding experimental barrier distributions for more ${ }^{7} \mathrm{Li}$-induced reactions.

\section{SUMMARY}

The quasielastic scattering excitation function at large backward angle has been measured for the system ${ }^{7} \mathrm{Li}+{ }^{159} \mathrm{~Tb}$ at energies around the Coulomb barrier and the corresponding barrier distribution has been extracted. The quasielastic scattering excitation function and the corresponding barrier distribution were determined, both with and without the contribution of $\alpha$ particles. The centroid of the quasielastic barrier distribution is seen to shift towards a higher energy with the inclusion of the contribution of $\alpha$ - particles. This corroborates the observations of Zagrabaev [9] for weakly bound systems.

The experimental "partial" quasielastic scattering cross sections and the barrier distribution determined only from the $\mathrm{Z}=3$ events have been compared with the coupled channel calculations. As proper bare potential was not available in the literature for the system ${ }^{7} \mathrm{Li}+{ }^{159} \mathrm{~Tb}$, the elastic scattering angular distribution data of Ref. 29] were re-analyzed in the CDCC framework to obtain a set of of properly adjusted cluster folded potentials for $\alpha+{ }^{159} \mathrm{~Tb}$ and $t+{ }^{159} \mathrm{~Tb}$ so as to reproduce the elastic scattering angular distribution data.

Using the cluster folded potentials for $\alpha^{159} \mathrm{~Tb}$ and $t$ ${ }^{159} \mathrm{~Tb}$ thus determined, the coupled channel calculations were done, including different couplings at a time, in the CDCC framework to see their effects on the measured quasielastic scattering excitation function and corresponding barrier distribution separately. The inelastic coupling scheme that included the low-lying excited states of ${ }^{159} \mathrm{~Tb}$ and the bound excited state of ${ }^{7} \mathrm{Li}$, fails to reproduce the experimental quasielastic excitation function and the barrier distribution for ${ }^{7} \mathrm{Li}+{ }^{159} \mathrm{~Tb}$. The quasielastic scattering excitation function could be reproduced reasonably well, except at the higher energies, by including coupling to the continuum of ${ }^{7} \mathrm{Li}$, in addition to the above inelastic couplings. Though the height of the quasielastic barrier distribution could be reproduced reasonably well, a small shift between the centroids of the experimental and theoretical barrier distribution was observed. The shift might be attributed to the effects of other reaction channels, especially transfer and transfer induced breakup processes not considered in the work.

As experimental $D_{\text {fus }}$ could not be obtained, the calculated fusion cross sections which reproduced the measured fusion cross sections of ${ }^{7} \mathrm{Li}+{ }^{159} \mathrm{~Tb} 24$ were used to derive the $D_{\text {fus }}$. The $D_{\text {fus }}$, thereby obtained was compared with the experimental $D_{q e l}$, both including and excluding the contribution of the $\alpha$-particles. This comparison indicates that the distribution $D_{q e l}$ including the contribution of $\alpha$-particles, is considerably similar to $\mathrm{CF} D_{\text {fus }}$ for ${ }^{7} \mathrm{Li}+{ }^{159} \mathrm{~Tb}$ system. Similar observation was also reported for ${ }^{7} \mathrm{Li}+{ }^{208} \mathrm{~Pb}$ [8], thus 
showing that the similarity between CF $D_{\text {fus }}$ and $D_{q e l}$, including $\alpha$-yield, might be independent of the structure of target nuclei. It has been argued that this similarity of $\mathrm{CF}$ barrier distribution with the barrier distribution obtained from the sum of quasielastic and $\alpha$-particle contributions, probably lies in the fact that the $t$-capture process is the dominant ICF process in ${ }^{7} \mathrm{Li}$-induced reactions with heavy mass targets. In order to have a better understanding of the role of $\alpha$-contributing channels on $D_{\text {fus }}$ and $D_{q e l}$ in reactions with weakly bound projectiles, more simultaneous measurements of fusion and quasielastic barrier distributions are needed for ${ }^{7} \mathrm{Li}$-induced reactions with various target nuclei, and also for reactions induced by other weakly bound projectiles.

\section{Acknowledgments}

We would like to thank the Pelletron crew for the smooth operation of the accelerator. Thanks are also due to Mr. Sujib Chandra Chatterjee of Saha Institute of Nuclear Physics for his technical support during the experiment.
[1] M. Beckerman, Phys. Rep. 129, 145 (1985)

[2] M. Dasgupta, D. J. Hinde, N. Rowley, A. M. Stefanini, Annu. Rev. Nucl. Part. Sci. 48, 401 (1998) and references therein

[3] N. Rowley, G.R. Satchler and P.H. Stelson Phys.A Lett. B254, 25 (1991)

[4] H. Timmers, J.R.Leigh, M.Dasgupta, D.J.Hinde, R.C.Lemmon, J.C.Mein, C.R.Morton, J.O.Newton, N.Rowley, Nucl. Phys. A584, 190 (1995)

[5] H. Timmers, D. Ackermann, S. Beghini, L. Corradi, J.H. He, G. Montagnoli, F. Scarlassara, A.M. Stefanini, and N. Rowley, Nucl. Phys. A633, 421 (1998)

[6] Huanqiao Zhang, Feng Yang, Chengjian Lin, Zuhua Liu and Yueming Hu, Phys. Rev. C57, 1047 (1998)

[7] R.F. Simoes, D.S. Monteiro, L.K. Ono, A.M. Jacob, J.M.B. Shorto, N. Added, E. Crema, Phys. Lett. B527, $187(2002)$

[8] C.J. Lin, H.Q. Zhang, F. Yang, M. Ruan, Z.H. Liu, Y.W. Wu, X.K. Wu, P. Zhou, C.L. Zhang, G.L. Zhang, G.P. An, H.M. Jia and X.X. Xu, Nucl. Phys. A787, 281c (2007)

[9] V.I. Zagrebaev, Phys. Rev. C78, 047602 (2008)

[10] S. Mitsuoka, H. Ikezoe, K. Nishio, K. Tsuruta, S.C. Jeong, and Y. Watanabe Phys. Rev. Letts. 99, 182701 (2007)

[11] S. S. Ntshangase,N. Rowley, R.A. Bark, S. V. Förtsch, J. J. Lawrie, E. A. Lawrie, R. Lindsay, M. Lipoglavsek, S. M. Maliage, L. J. Mudau, S. M. Mullins, O. M. Ndwandwe, R. Neveling, G. Sletten, F. D. Smit, and C. Theron, Phys. Letts. B 651, 27 (2007)

[12] D.S. Monteiro, O. A. Capurro, A. Arazi, J. O. Fernández Niello, J. M. Figueira, G. V. Martí, D. Martínez Heimann, A. E. Negri, A. J. Pacheco, V. Guimarães, D. R. Otomar, J. Lubian, and P. R. S. Gomes, Phys. Rev. C79, 014601 (2009)

[13] S. Mukherjee, B. K. Nayak, D. S. Monteiro, J. Lubian, P. R. S. Gomes, S. Appannababu, and R. K. Choudhury, Phys. Rev. C80, 014607 (2009)

[14] D. R. Otomar, J. Lubian, P. R. S. Gomes, D. S. Monteiro, O.A. Capurro, A. Arazi, J. O. Fernández Niello, J. M. Figueira, G. V. Martí, D. Martínez Heimann, A. E. Negri, A. J. Pacheco, V. Guimarães, and L. C. Chamon, Phys. Rev. C80, 034614 (2009)

[15] K. Zerva, N. Patronis, A. Pakou, N. Alamanos, X.
Aslanoglou, D. Filipescu, T. Glodariu, M. Kokkoris, M. La Commara, A. Lagoyannis, M. Mazzocco, N.G. Nicolis, D. Pierroutsakou, M. Romoli, and K. Rusek, Phys. Rev. C80, 017601 (2009)

[16] K. Zerva, A. Pakou, K. Rusek, N. Patronis, N. Alamanos, X. Aslanoglou, D. Filipescu, T. Glodariu, N. Keeley, M. Kokkoris, M. La Commara, A. Lagoyannis, M. Mazzocco, N. G. Nicolis, D. Pierroutsakou, and M. Romoli, Phys. Rev. C82, 044607 (2010)

[17] H.M. Jia, C.J. Lin, H.Q. Zhang, Z.H. Liu, N. Yu, F. Yang, F. Jia, X.X. Xu, Z.D. Wu, S.T. Zhang, and C.L. Bai, Phys. Rev. C82, 027602 (2010)

[18] K. Zerva, A. Pakou, N. Patronis, P. Figuera, A. Musumarra, A. Di Pietro, M. Fisichella, T. Glodariu, M. La Commara, M. Lattuada, M. Mazzocco, M.G. Pellegriti, D. Pierroutsakou, A.M. Sanchez-Benitez, V. Scuderi, E. Strano, and E. Rusek, Eur. Phys. J. A48, $102(2012)$

[19] M. Zadro, P. Figuera, A. Di Pietro, M. Fisichella, M. Lattuada, T. Lönnroth, M. Milin, V. Ostashko, M.G. Pellegriti, V. Scuderi, D. Stanko, E. Strano, and D. Torresi, Phys. Rev. C87, 054606 (2013)

[20] C.S. Palshetkar, Shital Thakur, V. Nanal, A. Shrivastava, N. Dokania, V. Singh, V. V. Parkar, P. C. Rout, R. Palit, R. G. Pillay, S. Bhattacharyya, A. Chatterjee, S. Santra, K. Ramachandran, and N. L. Singh, Phys. Rev. C89, 024607 (2014)

[21] Md. Moin Shaikh, Subinit Roy, S. Rajbanshi, M. K. Pradhan, A. Mukherjee, P. Basu, S. Pal, V. Nanal, R. G. Pillay, and A. Shrivastava, Phys. Rev. C91, 034615 (2015)

[22] Md. Moin Shaikh, Subinit Roy, A. Mukherjee, A. Goswami, Balaram Dey, S. Pal, S. Roy, A. Shrivastava, S.K. Pandit, and K. Mahata, Phys. Rev. C102, 024627 (2020)

[23] A. Mukherjee, Piyasi Biswas, Md. Moin Shaikh, Subinit Roy, A. Goswami, M.K. Pradhan, P. Basu, S. Santra, S.K. Pandit, K. Mahata, and A. Shrivastava, EPJ Web of Conferences 163, 00039 (2017)

[24] A. Mukherjee, Subinit Roy, M. K. Pradhan, M. Saha Sarkar, P.Basu, B. Dasmahapatra, T. Bhattacharya, S. Bhattacharya, S.K. Basu, A. Chatterjee, V. Tripathi, S. Kailas, Phys. Lett. B636, 91 (2006)

[25] M. K. Pradhan, A. Mukherjee, P. Basu, A. Goswami, 
R. Kshetri, Subinit Roy, P. Roy Chowdhury, M. Saha Sarkar, R. Palit, V.V. Parkar, S. Santra, and M. Ray, Phys. Rev. C83, 064606 (2011)

[26] M. K. Pradhan, A. Mukherjee, Subinit Roy, P. Basu, A. Goswami, R. Kshetri, R. Palit, V.V. Parkar, M. Ray, M. Saha Sarkar, and S. Santra, Phys. Rev. C88, 064603 (2013)

[27] S.K. Pandit, A. Shrivastava, K. Mahata, N. Keeley, V.V. Parkar, , Phys. Rev. C93, 061602 (2016)

[28] I. J. Thompson, Comput. Phys. Rep. 7, 167 (1988) and www.fresco.org.uk

[29] D. Patel, S. Mukherjee, D. C. Biswas, B. K. Nayak, Y. K. Gupta, L. S. Danu, S. Santra, and E. T. Mirgule, Phys. Rev. C91, 054614 (2015)

[30] A. Gómez-Camacho, J.Phys.: Conf. Ser. 730, 012012 (2016)

[31] D. Chattopadhyay, S. Santra, A. Pal, A. Kundu, K. Ramachandran, R. Tripathi, B.J. Roy, Y. Sawant, B.K. Nayak, A. Saxena, and D. Kailas, Phys. Rev. C98,
014609 (2018)

[32] http://www.nndc.bnl.gov

[33] R. Broda, M. Ishihara, B. Herskind, H. Oeschler, S. Ogaza, and H. Ryds, Nucl. Phys. A248, 356 (1975)

[34] K. Hagino, N. Rowley, and A.T. Kruppa, Comput. Phys. Commun. 123, 143 (1999)

[35] V.V. Parkar, Sushil K. Sharma, R. Palit, S. Upadhyaya, A. Shrivastava, S.K. Pandit, K. Mahata, V. Jha, S. Santra, K. Ramachandran, T.N. Nag, P.K. Rath, Bhushan Kanagalekar, and T. Trivedi, Phys. Rev. C97,014607 (2018)

[36] S.K. Pandit, A. Shrivastava, K. Mahata, V. V. Parkar, R. Palit, N. Keeley, P.C. Rout, A. Kumar, K. Ramachandran, S. Bhattacharyya, V. Nanal, C.S. Palshetkar, T.N. Nag, Shilpi Gupta, S. Biswas, S. Saha, J. Sethi, P. Singh, A. Chatterjee, and S. Kailas, Phys. Rev. C96, 044616 (2017) 